\title{
HiCEnterprise: Identifying long range chromosomal contacts in HiC data
}

\author{
Hania Kranas ${ }^{\text {Equal first author, 1, } 2 \text {, Irina Tuszyńska }}{ }^{\text {Equal first author, 1 , Bartek Wilczynski }}{ }^{\text {Corresp. } 1}$ \\ 1 Institute of Informatics, University of Warsaw, Warsaw, Poland \\ ${ }^{2}$ Current address: Institute for Research in Biomedicine (IRB Barcelona), The Barcelona Institute of Science and Technology, Barcelona, Spain \\ Corresponding Author: Bartek Wilczynski \\ Email address: bartek@mimuw.edu.pl
}

Motivation: Computational analysis of chromosomal contact data is currently gaining popularity with therapid advance in experimental techniques providing access to a growing body of data. An importantproblem in this area is the identification of long range contacts between distinct chromatin regions. Suchloops were shown to exist at different scales, either mediating relatively short-range interactions betweenenhancers and promoters or providing interactions between much larger distant chromosome domains. Aproper statistical analysis as well as availability to a wide research community are crucial in a tool for thistask . Results:We present HiCEnterprise, a first freely available software tool for identification of long rangechromatin contacts not only between small regions on, but also between chromosomal domains. Itimplements four different statistical tests for identification of significant contacts for user defined regionsor domains as well as necessary functions for input, output and visualization of chromosome contacts. 


\title{
HiCEnterprise: Identifying long range chromosomal contacts in HiC data
}

\author{
3 Hania Kranas ${ }^{1,2 *}$, Irina Tuszyńska ${ }^{1 *}$, and Bartek Wilczyński ${ }^{1}$
}

\author{
${ }^{1}$ Faculty of Mathematics, Informatics and Mechanics, University of Warsaw, Poland \\ ${ }^{2}$ Current address: Institute for Research in Biomedicine (IRB Barcelona), The Barcelona \\ Institute of Science and Technology, Baldiri Reixac, 10, 08028 Barcelona, Spain \\ *These authors contributed equally to this work
}

\author{
Corresponding author: \\ Bartek Wilczyński ${ }^{1}$ \\ Email address: bartek@mimuw.edu.pl
}

\section{ABSTRACT}

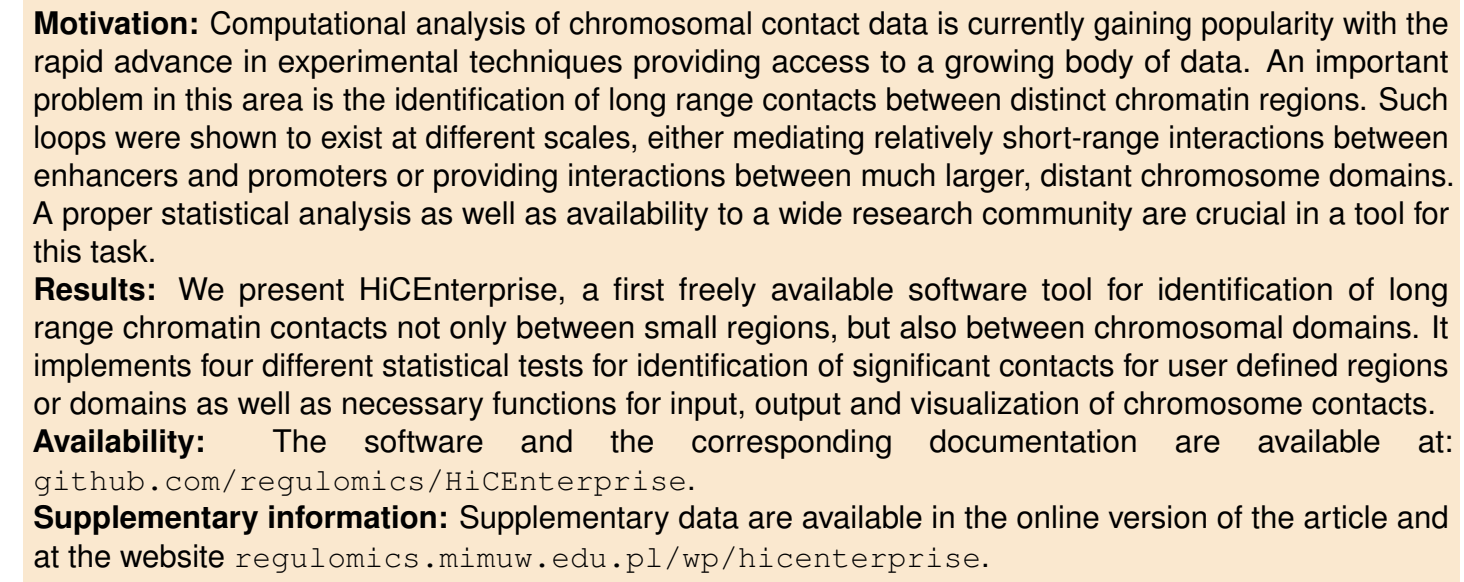
rapid advance in experimental techniques providing access to a growing body of data. An important problem in this area is the identification of long range contacts between distinct chromatin regions. Such loops were shown to exist at different scales, either mediating relatively short-range interactions between enhancers and promoters or providing interactions between much larger, distant chromosome domains. A proper statistical analysis as well as availability to a wide research community are crucial in a tool for this task.

Results: We present HiCEnterprise, a first freely available software tool for identification of long range chromatin contacts not only between small regions, but also between chromosomal domains. It implements four different statistical tests for identification of significant contacts for user defined regions or domains as well as necessary functions for input, output and visualization of chromosome contacts.

Availability: The software and the corresponding documentation are available at: github.com/regulomics/HiCEnterprise.

Supplementary information: Supplementary data are available in the online version of the article and at the website regulomics.mimuw.edu.pl/wp/hicenterprise.

\section{INTRODUCTION}

Chromosomes in eukaryotic cells are very complex assemblies of nucleic acids and proteins that function in a tightly packed 3D environment of the cell nucleus (Sazer and Schiessel, 2018). The packing of chromosomes is at the same time dynamic and visibly different between cells in the same population, yet its conformation is proven to be non-random to allow for efficient activation and repression of subsets of genes defined by the dynamic epigenetic state of the cell (Spector, 2003). Scientists have been interested in studying the rules governing the chromatin structure and its dynamics for a long time, however we were mostly limited to theoretical studies based on relatively sparse imaging data until the development of the Hi-C technique (Lieberman-Aiden et al., 2009). The body of data from Hi-C experiments is quickly growing, allowing us to answer more questions related to chromosome structure and its relation to gene regulation. In particular, the question of identifying chromosomal contacts have been studied both on the level of small regions (even down to $1 \mathrm{~kb}$ ) that could represent enhancer-promoter interactions (Won et al., 2016), as well as on the level of larger ones, like TAD-to-TAD interactions (Niskanen et al., 2017). While the enhancer-promoter interactions are widely studied, the importance of larger domain-domain interactions are not quite as well studied, however there are reports of important examples of such interactions having a regulatory function such as the polycomb related strong interaction in Drosophila(Sexton et al., 2012) or the cell-type-specific silencing interaction on human chromosome 17 (Niskanen et al., 2017). Even though a number of methods for chromatin contact detection were proposed, such as HOMER (Heinz et al., 2010), HiCCUPS(Rao et al., 2014), GotHIC (Mifsud et al., 2015) or Fit-Hi-C (Ay et al., 2014; Kaul et al., 2020) (see Forcato et al. (2017) for a review), there are still no freely 
A
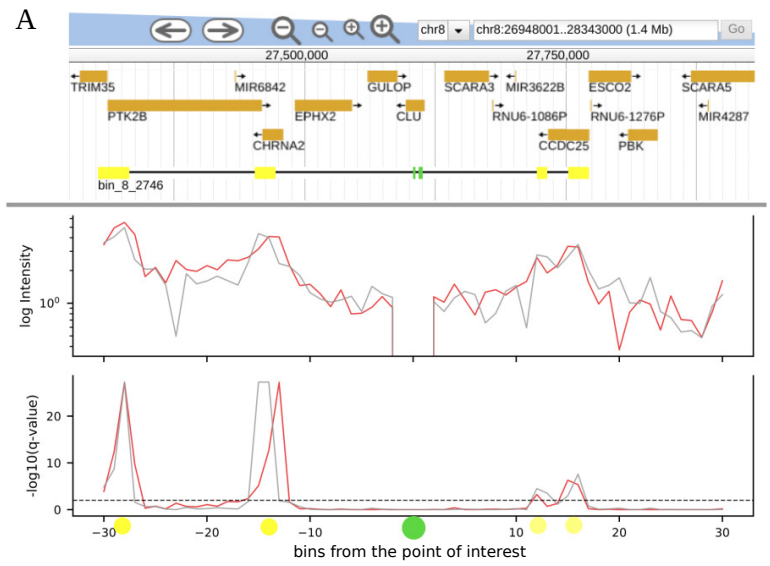

B

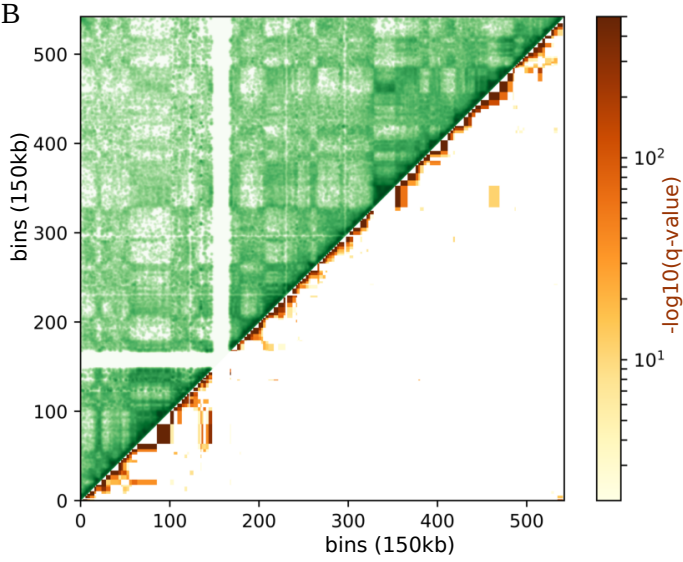

Figure 1. Example of visualization of long range contacts for: (a) regions, here enhancer-promoter contacts, for two $10 \mathrm{~kb}$ resolution Hi-C maps from human Fetal Brain cells. Contacts were calculated up to 50 bins distance each way from the point of interest. Top to bottom: a Jbrowse (Skinner et al., 2009) screenshot with genes and interaction profile representation (points of interest in green, their contact predictions in yellow); HiCEnterprise interaction profile plot with intensities (weighted by distance) and $-\log _{10}$ of FDR corrected p-values (q-values) with a threshold set at 0.01. (b) TADs, here HiCEnterprise visualization for $150 \mathrm{~kb}$ resolution Hi-C map of 17th chromosome from HUVEC. Left upper trianle: original Hi-C map contact frequencies; right lower triangle: $-\log _{10}$ of q-values of the interdomain interactions calculated with hypergeometric distribution.

available software tools for identification of those interactions between the larger segments, i.e. domains (the only available method, PSYCHIC (Ron et al., 2017) requires Matlab). Since more researchers are interested in identifying such contacts, it may be helpful for them to have a freely available implementation of statistical protocols that were adapted by us to identify long range chromatin interactions not only between points but also between chromosomal domains.

\section{METHODS}

HiCEnterprise is a package consisting of two types of contact analyses: between short regions (1-3 bins of a Hi-C map) and between topologically associating domains (TADs) (Pombo and Dillon, 2015). Here regions are short genomic sequences, of length between 1-3 bins, where a bin represents the smallest DNA segment analyzed given the Hi-C map resolution. TAD is defined as a larger segment, within which the DNA sequences physically interact with each other more frequently than with sequences outside of it. TADs can be noticed on the Hi-C map as dark (contact-rich) triangles near the diagonal.

The first part of the package, HiCEnterprise regions is used for identification of statistically significant long range contacts between small regions. The analysis implemented in this part is based on the method for identification of bin-to-bin interactions and creating interaction profiles based on Hi-C data as introduced by Won et al. (2016). As Hi-C cis-contact maps should be symmetrical, interaction profile for a region located in a particular bin is obtained by extracting intensities only horizontally, from the left and right of the region of interest positions on the diagonal. Significant contacts between bins are identified as enrichments under background distribution (fitted Weibull distribution matched by chromosome and distance, Won et al. (2016)). False Discovery Rate (FDR) calculated with a BenjaminiHochberg procedure (Benjamini and Hochberg, 1995) is used to correct for multiple testing, giving us $\mathrm{q}$-values in addition to the $\mathrm{p}$-values of every potential interaction.

It still may be difficult to distinguish between true interactions and noise, so to provide additional test for the validity of found interactions, we offer an option for simultaneous analysis of multiple biological replicates. If several Hi-C maps (replicates) are provided on input, interactions are considered significant only if their FDR value is below the selected threshold in all replicates. In the example interaction profile plot (Figure 1a) one can see the analysis for two Fetal Brain maps (Won et al., 2016), where for two enhancers located within one 10kb bin, 4 significant interactions with potential genes of interest have 
been found, confirmed by two replicate maps. The results shown are consistent with the results from the original paper (Won et al., 2016).

To run this point interactions analysis, it is required that the user provides at least one $\mathrm{Hi}-\mathrm{C}$ chromosome map in the numpy format and a BED file with coordinates of regions to extract interaction profiles. Since enhancers are distal regulatory elements that affect transcription levels of target genes by being in physical close proximity to them, an example use of the HiCEnterprise region mode would be to find DNA regions frequently in contact with particular enhancers based on Hi-C maps. As this mode will be likely often used exactly for the purpose of scanning for enhancer related interactions, we have provided also a mode in which the user can supply as input a list of enhancers from a FASTA file obtained from EnhancerAtlas (Gao et al., 2016), a database that provides annotation of enhancers in the human genome). HiCEnterprise can plot the results related with found interactions either with matplotlib (Hunter, 2007) or rpy2 (Gautier, 2012). Output files with found interacting regions are available in three formats: txt, BED-like and GFF-like. Remapping between assemblies is possible for BED and GFF files (pyliftover ${ }^{1}$ package is required).

The second part of HiCEnterprise, HiCEnterprise domains is dedicated to calculation of long range interactions significance scores for pairs of larger chromosome segments, like the topologically associating domains (TADs) in Hi-C contact maps, as introduced in (Niskanen et al., 2017). In this mode the user needs to define borders of TADs obtained using external software (e.g. HOMER by Heinz et al. (2010); see Dali and Blanchette (2017) for more examples). As the first step of this method, the new matrix $\mathrm{M}$ (with the shape of $\mathrm{NxN}$ where $\mathrm{N}$ is the number of domains in the chromosome considered) is calculated. $\mathrm{M}[\mathrm{i}, \mathrm{j}]$ represents the total number of $\mathrm{Hi}-\mathrm{C}$ contacts for the pair of domains $\mathrm{i}$ and $\mathrm{j}$. Next, for each pair of domains in the new matrix, a p-value is calculated based on the hypergeometric, Poisson or negative binomial test.

Our software calculates the parameters of the chosen distribution based on the data observed in the actual Hi-C matrix and calculates a p-value for enrichment under the null model. Similarly to the point analysis, p-values are converted to FDR q-values to account for multiple hypothesis testing. HiCEnterprise domains mode, that calculates the probability of inter domain contacts based on $\mathrm{Hi}-\mathrm{C}$ maps, can be run with different options. Some of them are necessary to run the program: the chromosome number, the Hi-C map, the resolution of Hi-C map, the file with domain borders information and the domain level if a hierarchical TAD caller like Sherpa (Krolak and Wilczynski, 2012) was used to determine the domain borders. It is possible to modify the threshold of the q-values that will be considered as significant and returned in the output file (the default value is 0.01). By default, the resulting $\mathrm{p}$ - and q-values are written to two text files. The user can also choose to make a plot with results as a contact map, with significant contacts between domains highlighted with color (as seen in Figure 1b). One can also change colors of the contact maps and/or interactions on the figure, propose the title or change the distance between ticks on the generated figures.

\section{RESULTS}

Unlike the native structure from crystallographic studies in the case of proteins or small nucleic acid molecules, chromatin has no native structure(Hajjoul et al., 2013). This makes the task of identifying domain contacts more subjective. So far, there is no gold standard to compare methods that predict the interactions of chromatin to, so it is only possible to compare available methods between each other. We compared the results of HiCEnterprise regions mode, HiCCUPS (Rao et al., 2014) and Homer (Heinz et al., 2010) between each other and did a short quantitative analysis.

For this task, we used human umbilical vein endothelial cells (HUVEC) Hi-C maps with $25 \mathrm{~kb}$ resolution, that were kindly provided by Henri Niskanen (described in our joint publication Niskanen et al. (2017)). We compared the interactions found by the aforementioned tools within +/- 100 bins around the region of interest for human chromosome 1. First, we found interactions by HiCCUPS and HOMER: HiCCUPS found interactions for 262 regions while Homer for 204 regions. Next, we combined HiCCUPS and Homer results and removed the duplicate regions to prepare an input file with regions of interest for HiCEnterprise regions mode. Our tool found interactions for 386 out of 396 given regions. Some regions of interest are indicated as interacting with more than one site, so we compared all possible interactions (1446 for HiCEnterprise, 283 for HiCCUPS and 209 for Homer) and found out

\footnotetext{
${ }^{1}$ https://pypi.org/project/pyliftover/
} 

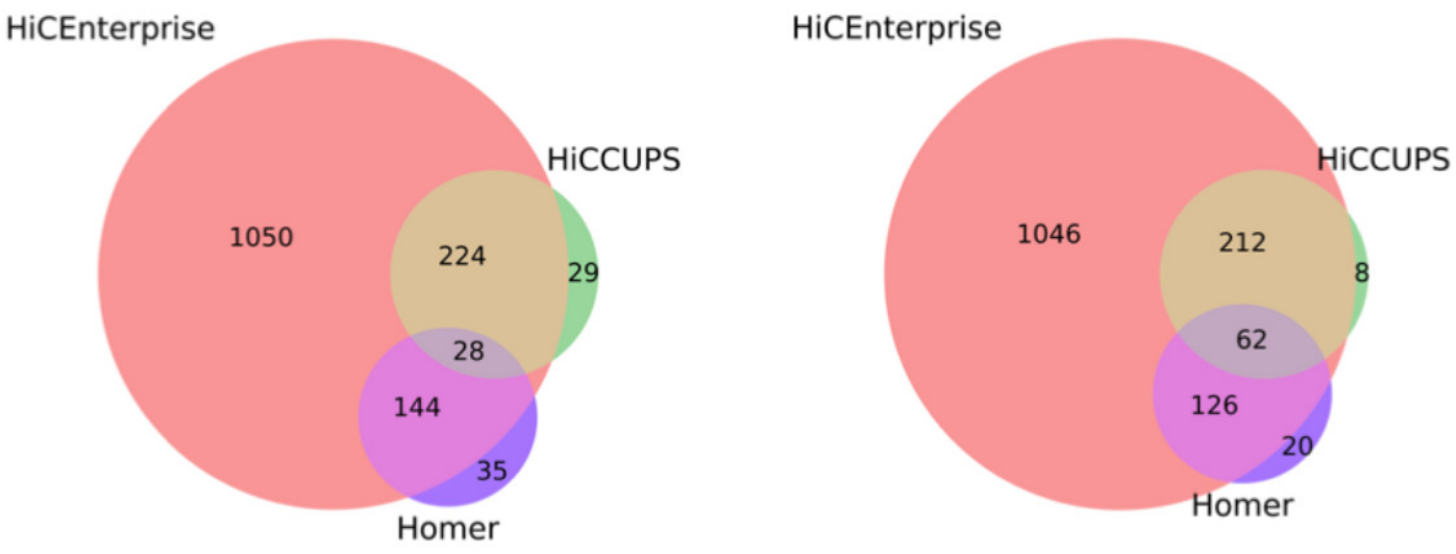

Figure 2. Left: Venn plot for comparing results intersection of HiCEnterprise with HiCCUPS and Homer programs. Right: Venn plot for comparing results intersection of HiCEnterprise with HiCCUPS and Homer programs with one bin freedom (if programs found the same bin $+/-1$, the contact is considered as common).

that HiCEnterprise recognised almost all contacts that were found by HiCCUPS and Homer programs, and proposed many additional statistically significant interaction places (Figure 2, left). Summing up, for 252 regions HiCEnterprise and HiCCUPS found the same interaction bin on the Hi-C map (p-value using binomial test $=3.17 * 10^{-295}$, while HiCEnterprise and Homer found the same bins of HiC map for 172 regions with p-value $1.21 * 10^{-171}$, whereas HiCCUPS and Homer found 28 identical interaction sites with p-value $7.49 * 10^{-30}$.

Sometimes, two methods found interactions between neighboring bins, differing just by 1 position on the chromosome, which may be explained as identification of a similar interaction, given that the enrichments are frequently spanning more than one bin. We checked how often this is the case, treating predicted interactions from different tools that fell within the $+/-1$ bin from each other as identical. As Homer had the smallest number of predicted interactions, we compared its results with the other two tools, and then checked common interactions for HiCCUPS and HiCEnterprise programs. With this approach, the commonalities between the three considered programs are even more obvious (Figure 2, right): we have 62 common interactions instead of 28 , and there are even fewer individual interactions found either only by HiCCUPS ( 8 instead of 29 ) or by Homer (20 instead of 35 ).

As HiCEnterprise regions-mode found many more significant interactions than the other tools we tested, we wanted to see how confident we can be in the functionality of those predictions. Thus, we overlapped the found interacting points from the aforementioned analysis (not the points of interest) with CTCF and RAD21 (cohesin component) Chip-seq peaks from HUVEC cells, provided by Henri Niskanen (Niskanen et al., 2017). We discovered that those interactions are significantly enriched over all queried bins in CTCF (chromatin binding factor) and RAD21 (cohesin complex component protein) Chip-seq peaks (p-value using binomial test $=1.16 * 10^{-71}, 8.27 * 10^{-75}$ accordingly). Additionally, we looked at how many of the predicted HiCEnterprise region-wise interactions are forming longer stretches of consecutive predictions. For the total of 1446 unique origin-interaction bins found, after clustering, we obtain 849 clusters of consecutive interactions. While most of those clustered interactions are constrained to up to 3 bins (probably due to the interacting region lying somewhere in-between those bins), we also find few very long interactions, up to 9 bins. We think that those might be reflection of larger, domain-wise interactions, however, thanks to the statistical model we employed here, the larger domain-wise interactions are not affecting the region-wise results in a significant manner.

To the best of our knowledge, there is no other freely available method for prediction of TAD-TAD interactions based on Hi-C maps that we could compare to. However, we wanted to understand if domains from one chromosomal compartment interact with domains in the same compartment more often than expected by chance. Chromatin compartments A and B, are usually defined by the first principal component (PC1) from Principal Component Analysis (PCA) - PC1 positive and negative values 
describes the A (active and accessible) and B (passive and closed) chromatin compartments accordingly (Lieberman-Aiden et al., 2009).

We started this analysis by detecting domain borders using Sherpa algorithm (https://github.com/regulomics/sherpa) developed by our group for the same 25kb HUVEC Hi-C map of chromosome 1 . Next, we calculated the probability of interactions between domain pairs using HiCEnterprise domain mode, using the three available distributions. We used PCA to identify the compartments to which the interacting domains belong. Each domain has been classified as belonging to a positive or negative compartment based on the number of positive or negative PC1 values within the domain. For all three methods, chi-square test rejected the null hypothesis according to which observed values would have the expected frequencies ( $\frac{n^{2}}{2}$ for negative-negative pairs and $\frac{p^{2}}{2}$ for for positive-positive, and $p * n$ for positive-negative, where $n \mathrm{i}$ the number of negative domains, and $p$ of positive). Next, binomial tests have shown the depletion of positive-negative pairs to be statistically significant (p-values $\leq 3.14 * 10^{-26}$, Figure S3) - which is in line with our expectation, as it has been shown that domains from one compartment tend to interact with domains from the same compartment.

Detailed description of all the analyses and results can be found in the Supplementary file HiCEnterprise_VS_HiCCUPS_Homer.pdf.

\section{DISCUSSION}

The software presented here is a flexible tool for identification of interacting loci based on Hi-C experiments, and the first freely available for calling domain-domain interactions. We provide two different functionalities (region-to-region and domain-to-domain contact identification) with several statistical tests that were already shown to be appropriate for each of the scenarios (Weibull distribution for regions and hypergeometric, Poisson and negative-binomial tests for domains analysis). This is in contrast to some earlier approaches like Fit-Hi-C that fit splines to the data or HiCCUPS that simply search for locally enriched regions in Hi-C maps.

HiCEnterprise regions mode is unique compared to other available tools - it finds statistically important interactions (pixels on the Hi-C map) between regions compared with other interactions at the same distance (in one diagonal of Hi-C map), HiCCUPS analyses the contacts between the regions of chromatin against the number of contacts in a series of regions surrounding the pixel, while Homer script identifies chromatin interactions by comparing local maxima with both local surrounding region and global interactions for the same genomic distance. Therefore it is in line with our expectation that programs that use different statistical models to calculate the interaction between regions on $\mathrm{HiC}$ maps not always found the same contact bins. However, in most cases HiCEnterprise found the same interactions as both HiCCUPS and Homer programs (Figure 2, left), while there are also regions, where programs found different contacts. Moreover, these point interactions found by HiCEnterprise are significantly enriched in CTCF and RAD21 Chip-seq peaks further suggesting their validity. Lastly, although there is no competing tool for the HiCEnterprise domain mode that we know of so far, we managed to show that the significantly interacting domain pairs are more often than expected belonging to the same chromosomal compartment.

In summary, the strength of our approach for both modes comes with assigning $\mathrm{p}$ - and q-values to Hi-C interactions, allowing the user to generate their own hypotheses, combined with additional functionalities that might help give additional evidence for the found interactions. By providing a tested and easy to use implementation, we hope to make it easier for experimentalists to use these methods without the need to implement them on their own.

\section{ACKNOWLEDGMENTS}

We would like to thank Minna Kaikkonen and Henri Niskanen from the University of East Finland for providing us with $\mathrm{Hi}-\mathrm{C}$ and ChIP-Seq data for testing purposes.

This work has been supported by the Polish National Science Center Grant decision number [DEC 2015/16/W/NZ2/00314].

\section{REFERENCES}

Ay, F., Bailey, T. L., and Noble, W. S. (2014). Statistical confidence estimation for hi-c data reveals regulatory chromatin contacts. Genome research, 24(6):999-1011. 
Benjamini, Y. and Hochberg, Y. (1995). Controlling the false discovery rate: a practical and powerful approach to multiple testing. Journal of the Royal statistical society: series B (Methodological), 57(1):289-300.

Dali, R. and Blanchette, M. (2017). A critical assessment of topologically associating domain prediction tools. Nucleic acids research, 45(6):2994-3005.

Forcato, M., Nicoletti, C., Pal, K., Livi, C. M., Ferrari, F., and Bicciato, S. (2017). Comparison of computational methods for hi-c data analysis. Nature methods, 14(7):679.

Gao, T., He, B., Liu, S., Zhu, H., Tan, K., and Qian, J. (2016). EnhancerAtlas: a resource for enhancer annotation and analysis in 105 human cell/tissue types. Bioinformatics (Oxford, England), 32(23):35433551.

Gautier, L. (2012). rpy2: A simple and efficient access to $\mathrm{r}$ from python, 2012. http://rpy. sourceforge. net/rpy2. html.

Hajjoul, H., Mathon, J., Ranchon, H., Goiffon, I., Mozziconacci, J., Albert, B., Carrivain, P., Victor, J.-M., Gadal, O., Bystricky, K., et al. (2013). High-throughput chromatin motion tracking in living yeast reveals the flexibility of the fiber throughout the genome. Genome research, 23(11):1829-1838.

Heinz, S., Benner, C., Spann, N., Bertolino, E., Lin, Y. C., Laslo, P., Cheng, J. X., Murre, C., Singh, H., and Glass, C. K. (2010). Simple combinations of lineage-determining transcription factors prime cis-regulatory elements required for macrophage and b cell identities. Molecular cell, 38(4):576-589.

Hunter, J. D. (2007). Matplotlib: A 2d graphics environment. Computing In Science \& Engineering, 9(3):90-95.

Kaul, A., Bhattacharyya, S., and Ay, F. (2020). Identifying statistically significant chromatin contacts from hi-c data with fithic2. Nature Protocols, 15(3):991-1012.

Krolak, K. and Wilczynski, B. (2012). Sherpa: Simple hierarchical profile aggregation. https://github.com/regulomics/sherpa.

Lieberman-Aiden, E., Van Berkum, N. L., Williams, L., Imakaev, M., Ragoczy, T., Telling, A., Amit, I., Lajoie, B. R., Sabo, P. J., Dorschner, M. O., et al. (2009). Comprehensive mapping of long-range interactions reveals folding principles of the human genome. science, 326(5950):289-293.

Mifsud, B., Tavares-Cadete, F., Young, A. N., Sugar, R., Schoenfelder, S., Ferreira, L., Wingett, S. W., Andrews, S., Grey, W., Ewels, P. A., et al. (2015). Mapping long-range promoter contacts in human cells with high-resolution capture hi-c. Nature genetics, 47(6):598.

Niskanen, H., Tuszynska, I., Zaborowski, R., Heinäniemi, M., Ylä-Herttuala, S., Wilczynski, B., and Kaikkonen, M. U. (2017). Endothelial cell differentiation is encompassed by changes in long range interactions between inactive chromatin regions. Nucleic acids research.

Pombo, A. and Dillon, N. (2015). Three-dimensional genome architecture: players and mechanisms. Nature Reviews Molecular Cell Biology, 16(4):245-257.

Rao, S. S., Huntley, M. H., Durand, N. C., Stamenova, E. K., Bochkov, I. D., Robinson, J. T., Sanborn, A. L., Machol, I., Omer, A. D., Lander, E. S., et al. (2014). A 3d map of the human genome at kilobase resolution reveals principles of chromatin looping. Cell, 159(7):1665-1680.

Ron, G., Globerson, Y., Moran, D., and Kaplan, T. (2017). Promoter-enhancer interactions identified from hi-c data using probabilistic models and hierarchical topological domains. Nature communications, $8(1): 2237$.

Sazer, S. and Schiessel, H. (2018). The biology and polymer physics underlying large-scale chromosome organization. Traffic, 19(2):87-104.

Sexton, T., Yaffe, E., Kenigsberg, E., Bantignies, F., Leblanc, B., Hoichman, M., Parrinello, H., Tanay, A., and Cavalli, G. (2012). Three-dimensional folding and functional organization principles of the drosophila genome. Cell, 148(3):458-472.

Skinner, M. E., Uzilov, A. V., Stein, L. D., Mungall, C. J., and Holmes, I. H. (2009). JBrowse: a next-generation genome browser. Genome Research, 19(9):1630-1638.

Spector, D. L. (2003). The dynamics of chromosome organization and gene regulation. Annual review of biochemistry, 72(1):573-608.

Won, H., de La Torre-Ubieta, L., Stein, J. L., Parikshak, N. N., Huang, J., Opland, C. K., Gandal, M. J., Sutton, G. J., Hormozdiari, F., Lu, D., et al. (2016). Chromosome conformation elucidates regulatory relationships in developing human brain. Nature. 